Paul Raymont (praymont@ryerson.ca or paulraymont@hotmail.com) Published in “On Causal Relevance: A Reply to Sullivan”, Dialogue (Canadian Philosophical Review), XLIII (2004): 367-76.

\title{
On Causal Relevance: A Reply to Sullivan
}

Paul Raymont carleton university

I want to thank Arthur Sullivan for his response (this issue) to my article, "Are Mental Properties Causally Relevant?" (Raymont 2001). In that article, I argued that much of the recent debate about epiphenomenalism invokes a confused notion of causal relevance. While Sullivan also finds fault with this debate, he does so for different reasons, and is not convinced by my critique to abandon the notion of causal relevance.

My response is complicated by the fact that I now believe that there is a notion of causal relevance that is not threatened by the argument that I offered in the final section of my (2001). I there argued that a prevalent notion of causal relevance is confused because it encapsulates mutually incompatible causal and explanatory requirements. Sullivan finds this critique convincing (Sullivan 2004, p. 5), but believes that the notion of causal relevance can be recuperated by purging it of any explanatory requirement. ${ }^{1} \mathrm{I}$ have recently moved in a different direction (Raymont 2003), aiming to salvage a notion of causal relevance by weakening the explanatory requirement to which it is subject (and arguing that on this weaker reading there is no reason to doubt the causal relevance of mental properties). Nevertheless, even though I no longer impose the stronger explanatory requirement on causal relevance, I will argue that Sullivan's case against that requirement is flawed. I will also argue that part of Sullivan's critique is in fact directed against the weaker explanatory requirement on causal relevance, one that should be preserved. 
The notion of causal relevance, as it has evolved in the literature, carries an explanatory requirement according to which something is causally relevant to another thing only if the former explains the latter. For instance, the loudness of the gun's firing is not causally relevant to the fatality because the loudness does not explain the fatality; and the redness of the brick is not causally relevant to the window's breaking because it does not explain that event. In such cases, we refer to the cause in a way that does not explain the effect. We explain the effect only when we consider the cause under the right description. I argued (Raymont 2001, pp. 520-23) that it is this possibility for unexplanatory citation of the cause that led to the introduction of the notion of causal relevance. But if it is thus an epistemological notion, the idea of causal relevance is also supposed to be metaphysical, since a causally relevant item is supposed to be a mindindependent, causally efficacious entity that plays a role in producing the effect. Thus conceived, the idea of causal relevance encapsulates mutually incompatible causal and explanatory requirements; the very notion represents a misguided attempt to reify the conception under which the cause is explanatory, projecting it as an entity in the cause that makes the cause produce its effect. In short, what people are after when they talk about causal relevance is at once a cause and an explaining of the effect, an efficacious item such that merely to introduce it is thereby to explain (Raymont 2001, p. 522). But there can be no such thing, for no degree of ontological augmentation in the cause will get us all the way to an explanation.

Sullivan presents the explanatory requirement as follows: “(An instance of) a property $\mathrm{F}$ cannot be causally relevant to (an instance of) a property $\mathrm{G}$ unless the presence of the instance of F explains the presence of the instance of G" (Sullivan 2004, 
p. 4). Here, causal relevance is a relation between property instances, but, despite appearances, explanation is not. In an endnote, Sullivan says that explanation is a relation between "conceptions of properties" (2004, p. 9, n. 6). This qualification brings to the fore the epistemological nature of the explanatory requirement.

Sullivan goes further, rendering explanation too subjective. He says, "Explanation is thoroughly knowledge- and interest-relative, and thus too amorphous to be criterial in deciding causal relevance" (2004, p. 6; emphasis added). In developing this claim, he ends up at the view that whether something really is an explanation is a function of whether someone 'finds', ${ }^{2}$ or believes, it to be such. This is evident from his example of the Medieval astronomers, who "saw" in their antiquated models "all manner of explanatory connections among the motions of celestial bodies" (Sullivan 2004, p. 6). Sullivan uses this example to argue that an explanatory connection between properties $\mathrm{F}$ and $\mathrm{G}$ is not sufficient for F's being causally relevant to G. But this only follows if his example of the Medieval astronomers is one in which there really is an explanatory connection. So Sullivan is here relying on the principle that there is an explanatory connection (among the properties so conceived) if someone believes there to be such a connection, or, in his terms, if someone 'finds' the connection to be explanatory (2004, pp. 6, 7 and 8). But this is false. As Sullivan notes $(2004$, p. 8) someone can go wrong (as the Medieval astronomers did) in 'finding' something to be explanatory; but then whether something is explanatory must be independent of whether people 'find' it to be explanatory-otherwise, there would be no possibility of being wrong in what one takes to be explanatory. If you are wrong in what you take to be explanatory, that can only be 
because what you found to be explanatory isn't. So explanation is not subjective in the way that Sullivan suggests. ${ }^{4}$

In my critique of causal relevance, the explanatory requirement should be interpreted in this less subjective way: when I said that a causally relevant item is supposed to be explanatory in the strong sense that merely to cite it is thereby to explain, 'explain' means really explain, not just be thought to explain. Real explanation is what people aim at when they seek a causally relevant item. ${ }^{5}$ And yet, as I argued (2001, pp. 520-23), nothing can be both a real explanation and an efficacious entity in the cause.

In an endnote, Sullivan challenges the requirement that causally relevant items should really be explanatory, and thus be what people ought to find explanatory (2004, p. 10 n. 8). I am not sure I understand his concern. He says that the requirement would "lack any real bite" (ibid.) since it would be too difficult to establish a real explanatory connection, for it would be too difficult "to rule out the possibility that we are mistaken" (ibid.). Granted, our judgments about what really is explanatory are fallible, but then so too are our judgments about what really is causally relevant. Indeed, in the sense in which the notion of causal relevance is used in the literature, it seems that to the extent that our judgments of what is explanatory are fallible, to precisely that extent our judgments about what is causally relevant are fallible; and this is because evidence that something is causally relevant just is evidence that it is a good explanation-at least, I do not see what other reason there could be for taking something to be causally relevant. This is all in keeping with the explanatory requirement, according to which something is causally relevant to an effect only if it is explanatory of it. In short, the mere fact that people can go wrong in their judgments about whether something is a good explanation in no way 
impugns the idea that what they aim for when they seek a causally relevant item is a good explanation.

Finally, if Sullivan aims at a notion of causal relevance that is not subject to an explanatory requirement, then he must show why that notion is not just that of causation with fine-grained relata (e.g., property instances). ${ }^{6}$

Again, I now think it possible to define a notion of causal relevance that is subject to a weaker explanatory requirement than the one that I considered in my (2001). I there claimed that the proponents of causal relevance seek something the mere description of which is guaranteed to explain. I said this based on the examples that are typically used to introduce the notion. For instance, in referring to the gunshot as a loud sound, I refer to the cause, but not in a way that accounts for the ensuing fatality. I inferred from such cases that what the proponents of causal relevance must be after is something that (unlike the cause) is guaranteed to do this explaining whenever we introduce it into our discourse, but that is also an entity in the cause that makes it produce the effect. It is clear that no entity can meet this demanding explanatory requirement, since anything we talk about can be talked about in an unexplanatory way (e.g., as the event referred to on p. 5 of a book). I inferred that to introduce the notion of causal relevance is to make no advance beyond those who, following Donald Davidson (1993), eschew causal relevance, ${ }^{7}$ preferring instead to speak simply of the cause (a dated particular that produces the effect) and explanations that refer to the cause.

Causal relevance need not be subjected to the demanding explanatory requirement with which I burdened it (of being guaranteed to explain once referred to). Causes cause what they do because of the way they are, that is, because of the properties they have, and 
this is all that is required by the notion that some properties of the cause are causally relevant. One may object that this simple, intuitive dictum involves a mistaken view of what does the causing; for it really is the event, not its properties or states of affairs involving them, that is the cause. This seems to be why Davidson (1993) denied that events cause their effects in virtue of their properties (which are then said to be 'causally relevant'). However, as Tim Crane has argued, this is unsatisfactory since it leaves us "unable to answer the question of why certain explanations are better than others by invoking the efficacious features of reality" (Crane 1995, p. 227; emphasis in the original). For example, the reason why we can explain the window's breaking by appealing to just some of the properties of its cause (e.g., its being a striking of the window by a brick with a given mass) is that those are the features of the cause in virtue of which it made the window break. Notice that in saying that it is because of some of its properties that the event produced the effect it is still the event that is being said to have caused the effect. So, contrary to Davidson's objection, we can allow for the idea of causally relevant properties without abandoning the view that events are the causal relata. $^{8}$ Moreover, the state of affairs (viz., the cause's having that mass), while it is not itself the cause of the window's breaking, is explanatory of this explanandum. And this is so even if we can appeal to this explanatory state of affairs without ourselves thereby explaining the effect of which it is explanatory. So the state of affairs and the property involved in it remain explanatory even though they can be cited in unexplanatory ways, that is, even though they do not meet the impossibly strong explanatory standard that I adopted in my (2001). Something is causally relevant only if it is explanatory in this weaker sense. 
This makes causal relevance metaphysical in the sense that it requires a mindindependent explanatory relation. This sounds similar to the idea of causal relevance that Sullivan endorses $(2004$, p. 5). However, while the causal relevance relation that I have sketched is not subject to the strong explanatory requirement (on which reference to causally relevant items is guaranteed to explain), it is still subject to a weaker one. The weaker requirement is this: property $\mathrm{F}$ is causally relevant to property $\mathrm{G}$ only if event c's having property $\mathrm{F}$ is explanatory of event e's having $\mathrm{G}$, in the sense that under some description of c's having F one can explain e's having G. So, where there is causal relevance there is something that, suitably described, supplies an explanation of the effect.

Sullivan's notion of causal relevance does not carry this weaker explanatory requirement. This is evident from his quantum mechanics example, which is supposed to show that explanatory connections are not necessary for causal relevance (2004, p. 6). There would be no need to resort to such exotic quantum cases if one intended to undermine just the strong explanatory requirement, for that requirement can be challenged well enough by means of a simpler, mundane case like the one involving the brick's breaking the window. To wit, the event in which the brick strikes the window causes the window's breaking, and it does so in virtue of some of its properties (e.g., the brick's mass) and not in virtue of some others (e.g., the brick's colour) $;^{9}$ the causally relevant properties account for the cause's production of its effect even though the way in which one refers to them (e.g., as Ed's favourite properties) is not guaranteed to explain the outcome. In fact, nothing about the person who tries to explain the outcome (e.g., how 
she refers to something) has any bearing on whether one state of affairs is in fact explanatory of the other.

Rather than make his point by means of some such ordinary case, Sullivan uses a more complicated illustration from quantum mechanics, the 'two-slit' case (Sullivan 2004, p. 6). Here, he says, I judge that some features of the initial conditions are causally relevant to what follows based on my knowledge of the data, which establish the requisite correlation. So there is causal relevance here. Nevertheless, I "lack something that is required to find these relations explanatory" (ibid.). Notice, though, that in his example it is not just that one does lack something required for finding the connection explanatory. If that were the point, then any old case in which one lacks an appropriate characterization of the causally relevant features, because (e.g.) one knows of them only as Ed's favourite features, would suffice. Instead, the point of the quantum example seems to be that one does not find the current characterization of things to be explanatory, and that there is no other way in which to characterize matters that would enable one to find the connection explanatory (assuming there are no hidden variables). There is nothing further in the case that, suitably described, would give us the explanation that we feel to be missing. So this example seems to be directed at the weaker explanatory requirement, for it is supposed to be a case in which there is causal relevance in the absence of any factors that, suitably described, would supply an explanation of the effect. That is, we seem to have a case in which the features that are causally relevant to the effect are so without being explanatory of it in even the weaker sense.

The example does not succeed in prising apart being causally relevant to something from being explanatory of it. After all, no causally explanatory connection 
will dispel the appearance of contingency in the relation between cause and effect, for no such explanatory link will give their relation the status of being knowable a priori. There will thus always remain the appearance of contingency in the link. In this way, as Hume said, causal explanation "only staves off our ignorance a little longer" about why it is that one sort of cause issues in a given type of effect (Hume [1748] 1977, IV.1, p. 19). One can agree without subscribing to Hume's treatment of the causal relation as a mere 'constant conjunction'. Make the causal relation modally stronger; it will still be true that one cannot discover what sort of effect will be produced by reflecting on the nature of the cause. There is no a priori link between the two. In that sense, there will always be a mystery concerning why one sort of thing should produce another (even if this is driven from our attention by the sense of familiarity that results from frequent experience of the relation). This is part of the mystery that is felt in the double-slit case. That case seems even more problematic because it violates Hume's 'spatial contiguity' condition for causation; but given enough familiarity with causal transactions that do not meet this condition, it should cease to exert such a strong influence on our intuitions concerning whether we have in fact got a causal explanation.

To the extent that one does feel that something explanatory is missing in the double-slit case, to that extent one will feel that something causally relevant is missing, or that there is a gap in the list of causally relevant features. To feel an explanatory gap is to feel a gap in causally relevant properties, features of the cause that would explain its production of the effect in the sense of being that 'in virtue of which' the cause gave rise to the effect. ${ }^{10}$ What we feel to be missing, then, are causally relevant features that would supply a fuller explanation, the absence of which leaves us so puzzled. Another way to 
see this is to ask what people do when they try to remedy the felt gap in explanation. They typically do so by postulating 'hidden variables', unobserved features that purport to explain the passage from the initial conditions to the observed effect; that is, they conjecture that there are more causally relevant factors on hand, items that, suitably described, would account for the effect. The search for hidden variables, then, is a search for features that are causally relevant to, and that therefore explain, the effect. If one sees no gap in the explanatory framework that needs filling by 'hidden variables', that is because one has come to believe that the already identified causally relevant properties supply a complete explanation of the effect. That is, to the extent that one is satisfied that these are all the causally relevant features required for the cause's production of the effect, to that extent one will feel that they are a full explanation of it. So I do not agree with Sullivan's description of the 'double-slit' case as one in which we are entitled to recognize a complete set of causally relevant features as such while not finding them to be explanatory. ${ }^{11}$ Either one really does see them as a complete set of causally relevant features, in which case one sees them as supplying a complete explanation, or one regards them as offering only a partial explanation and therefore as only an incomplete set of causally relevant features.

With this brief sketch of causal relevance before us, it is unclear why it should be thought to sustain doubts about the causal relevance of mental features. Indeed, contrary to what Sullivan suggests (2004, pp. 7-9), their relevance is compatible with the principle of the completeness of physics, even if that principle is formulated in terms of causal relevance. In its most plausible version, this principle amounts to the claim that every physical effect has a physical cause, the physical features of which are causally relevant 
to (hence explanatory of) its production of that effect; and the set of physical features is 'complete' in the sense that it does not require supplementation by non-physical properties in order to yield an account, without gaps, of why the cause produced that effect. Unlike the completeness principle that Sullivan considers (2004, p. 7), this does not mean that the physical features of the cause are its only causally relevant features. All that is required is that those physical features suffice for an explanation, and this does not rule out the possibility that other, higher-level features might also be causally relevant.

In order to rule out this possibility one must show that this superabundance of causally relevant features involves an unacceptable overdetermination. The prospects for doing so are dim. When one says that properties are causally relevant, one means that the cause produces its effect in virtue of those properties. The phrase 'in virtue of' introduces factors that figure in an explanation of the cause's production of its effect. So used, there is little reason to regard 'in virtue of' as introducing genuinely efficacious things. After all, a cause acts as it does in virtue of its time and place, but that is hardly a reason to treat places and times as being efficacious. So we do not multiply causes by multiplying causally relevant factors. There is still just the one event that is the cause. All that has been multiplied are ways of explaining why that single cause produced its effect. But if the context, 'in virtue of ...', does not here introduce efficacious things (e.g., property instances or 'tropes'), then intuitions about causation-notably, about causal overdetermination — should not be transferred to the supposedly distinct relation of causal relevance. To summarize, while it seems undeniable that an effect is produced by an event in virtue of its properties, it is unclear just what relation between the event and its properties is signified by 'in virtue of'. Whatever it is, it isn't a causal relation; a property 
does not cause the event that has it to cause an effect. Hence it is unclear why the causal relevance of properties should be thought to be a competitive relation, such that if we can account for the cause's efficacy by appeal to one of its properties, then no other feature of the cause can be causally relevant to the same outcome.

One might object that once we have explained something once, physically, there is no reason to explain it in a different way, by appeal to mental features. After all, what explanatory work is left for the mental to do? However, this is, at best, a point about the pragmatics of our speech acts of explaining. Having uncovered the full physical story about why a cause produced some behaviour, we may feel no need to explain it again. But that will not cause the explanatory connections between mental and behavioural features to dissolve, for surely those are objectively real (nomic or counterfactual) connections. Whether an explanatory connection obtains between features should not depend on whether we find it useful to consider it or refer to it in our speech acts of explaining.

If one is still troubled by the profligacy of causally relevant features, that is likely because one thinks it a puzzling coincidence that so many different features of the cause should be relevant to its efficacy. However, as long as there is some systematic dependence or supervenience of mental upon physical features, their co-instantiation in advance of the same sorts of effect should seem less puzzling. ${ }^{12}$

In view of these considerations, the onus is on those who doubt the causal relevance of the mental to define a conception of causal relevance on which the causal relevance of the physical can be seen to jeopardize that of the mental. ${ }^{13}$

\section{Notes}


1 “[T]he explanatory requirement has to go" (Sullivan 2004, p. 5).

${ }^{2}$ In this context, talk of 'finding' something to be explanatory is misleading if one treats 'finds' as a success verb, such that one can find $\mathrm{x}$ to be $\mathrm{F}$ only if $\mathrm{x}$ really is $\mathrm{F}$. As Sullivan notes (2004, p. 8), one can go wrong in 'finding' something to be explanatory. So I will continue to use quotes around 'finds', and will treat the locution 'finding' something to be explanatory as synonymous with believing (or taking) it to be explanatory.

${ }^{3}$ Since it too may be interpreted as a success verb, 'saw' also is misleading since one can be wrong in what one takes to be explanatory.

${ }^{4}$ Sullivan might distinguish between two ways in which one can go wrong in finding something to be explanatory. First, we may say that one set of statements explains an explanandum if the set bears the right inferential or supporting relation to the explanandum. In this sense, there may be several sets of statements that 'explain' the explanandum; for example, if we adopt the deductive-nomological model of explanation, there may be different (and even mutually incompatible) sets of statements, each one including a statement of a law and of initial conditions, and such that each one of these sets entails the explanandum. One can then go wrong in finding some set of statements to be explanatory by taking it to entail the explanandum when in fact it does not. This is the first way in which one can be wrong. (A similar point can be made in terms of other models of explanation, so the deductive-nomological model is not essential to my case.) I take it the Medieval astronomers have not made this sort of mistake. The statements that they take to be explanatory really are explanatory in this sense. However, among the many competing sets of statements that are explanatory in this sense, only one of them really is explanatory in the sense of reflecting the actual mechanisms 'out there in the 
world' that generate the effect that we are trying to explain. One can go astray by believing a proposed explanans to reflect these actual mechanisms when in fact it does not. This is the second way in which one can be mistaken in 'finding' something to be explanatory. It is presumably in this sense that Sullivan says the astronomers were wrong (2004, p. 8). This does not affect the point that we have a notion of something's really being explanatory of something else, which cannot be analyzed in terms of what one 'finds' to be explanatory.

${ }^{5}$ Sullivan says, "The quest for understanding some phenomena is a quest for identifying a minimal set of causally relevant properties" $(2004$, p. 6). But how could we hope to meet our aim of 'understanding' (i.e., explaining) something by identifying features that are causally relevant to it unless it were true that to identify the causally relevant features is thereby to explain the phenomenon under investigation? But to admit this much is to subscribe to an explanatory requirement on causal relevance.

${ }^{6}$ Cf. my 2001, pp. 515-17.

${ }^{7}$ Contrary to what Sullivan suggests (2004, p. 3), Davidson rejected any notion of causal relevance on which causes are thought to produce their effects in virtue of their properties.

${ }^{8}$ My thinking here is influenced by McLaughlin (1993), esp. pp. 30-32.

${ }^{9}$ Strictly speaking, the mass and colour are properties of the brick, but the event has properties corresponding to them (e.g., being a striking of a window by something with that mass). 
${ }^{10}$ This phrase, 'that in virtue of which', is often used to introduce the notion of causally relevant properties. I can see no way to interpret the phrase except as introducing an explanation.

${ }^{11}$ Where finding something to be explanatory does not remove the Humean sense of contingency.

${ }^{12}$ Cf. Block 1990.

${ }^{13}$ Thanks to Arthur Sullivan and to the anonymous referees for their comments. Support from a Social Sciences and Humanities Research Council of Canada Postdoctoral Fellowship is gratefully acknowledged.

\section{References}

Block, Ned

1990 “Can the Mind Change the World?" In Meaning and Method: Essays in Honor of Hilary Putnam. Edited by George Boolos. Cambridge, UK: Cambridge University Press.

Crane, Tim

1995 "The Mental Causation Debate." Proceedings of the Aristotelian Society (Suppl.), 69: 211-36.

Davidson, Donald

1993 “Thinking Causes.” In Mental Causation. Edited by John Heil and Alfred Mele. Oxford: Clarendon Press, pp. 3-17.

Hume, David

[1748] 1977 An Enquiry Concerning Human Understanding. Edited by Eric Steinberg. Indianapolis, IN: Hackett Publishing Company.

McLaughlin, Brian P.

1993 "On Davidson's Response to the Charge of Epiphenomenalism." In Mental Causation. Edited by John Heil and Alfred Mele. Oxford: Clarendon Press, pp. 27-40.

Raymont, Paul

2001 “Are Mental Properties Causally Relevant?” Dialogue, 40: 509-28. 
2003 "Kim on Closure, Exclusion and Nonreductive Physicalism." In Physicalism and Mental Causation. Edited by Sven Walter and HeinzDieter Heckmann. Exeter, UK: Imprint Academic, pp. 225-42.

Sullivan, Arthur

2004 “On Causal Relevance: a Reply to Raymont.” Dialogue, ??. 